\title{
Trigeminal nerve injuries in oral and maxillofacial surgery: a literature review
}

\author{
Matthew Schiavone^, Vincent B. Ziccardi \\ Department of Oral and Maxillofacial Surgery, Rutgers School of Dental Medicine, Newark, NJ, USA \\ Contributions: (I) Conception and design: Both authors; (II) Administrative support: VB Ziccardi; (III) Provision of study materials or patients: Both \\ authors; (IV) Collection and assembly of data: Both authors; (V) Data analysis and interpretation: Both authors; (VI) Manuscript writing: Both \\ authors; (VII) Final approval of manuscript: Both authors. \\ Correspondence to: Matthew Schiavone, DMD, MD; Vincent B. Ziccardi, DDS, MD, FACS. Department of Oral and Maxillofacial Surgery, Rutgers \\ School of Dental Medicine, 110 Bergen Street, Room B854, Newark, NJ 07103, USA. Email: mrs368@sdm.rutgers.edu; ziccarvb@sdm.rutgers.edu.
}

\begin{abstract}
Objective: Trigeminal nerve injury is an uncommon complication of outpatient oral surgery and dental procedures such as removal of impacted teeth, placement of endosseous dental implants, and even injection of local anesthesia. In the field of oral and maxillofacial surgery, the two most commonly injured branches of the trigeminal nerve are the inferior alveolar and lingual nerves. Fortunately, most nerve injuries will undergo spontaneous recovery, with only a small percentage of cases resulting in permanent sensory deficits.

Background: There is significant morbidity associated with trigeminal nerve injuries, however. Affected patients may complain of difficulty with speech, mastication, and dysesthesia among other symptoms. Once a nerve injury has been identified, the degree of injury is defined based on published classification systems such as the Medical Research Council Scale. Specific neurosensory testing can be performed in the office, and serial neurosensory testing is recorded in order to track a patient's progress over time. In cases with persistent symptoms, trigeminal nerve microsurgery may be indicated. Surgery entails isolating the affected nerve, removing adherent scar tissue, and occasionally excising a fibrotic portion of the nerve with direct neurorrhaphy. When a large continuity defect is present, a nerve graft may be indicated.

Methods: A comprehensive literature review was conducted on the etiology, diagnosis, and treatment of trigeminal nerve injuries. Multiple databases were used to find relevant articles, including PubMed and MEDLINE.

Conclusions: Trigeminal nerve injuries occur infrequently in oral and maxillofacial surgery, but such injuries can be worrisome for patients and providers alike. Thankfully, trigeminal nerve microsurgery has the potential to provide affected patients with improved functional sensory recovery and quality of life.
\end{abstract}

Keywords: Trigeminal; nerve; microsurgery

Received: 10 March 2021; Accepted: 25 July 2021; Published: 10 September 2021.

doi: 10.21037/fomm-21-26

View this article at: https://dx.doi.org/10.21037/fomm-21-26

\section{Introduction}

There are numerous procedures performed by oral and maxillofacial surgeons and dentists which can potentially injure the trigeminal nerve. A thorough understanding of the etiology and management of such injuries is imperative for oral surgeons to practice safer and more efficiently in both the office and hospital settings. Third molar removal represents the most common etiology of injury (1). The injury rate for the inferior alveolar nerve (IAN) has frequently been cited as 4 per 1,000 mandibular third

\footnotetext{
^ ORCID: 0000-0003-4879-0446.
} 
molar extractions (1). The rate for lingual nerve injury is lower, at approximately 1 per 1,000 mandibular third molar extractions (1). As many as $25 \%$ of these patients may have persistent sensory deficits up to one year following the time of injury (2). Prior to third molar extractions, it is incumbent upon the surgeon to have a thorough discussion with the patient regarding the risks of the procedure, and informed consent must be obtained. Despite the informed consent process, these injuries still represent a significant source of litigation against clinicians (3).

Lingual nerve injuries are particularly devastating, as they may cause difficulty with speech, taste, and masticatory function (3). The lingual nerve exhibits a tremendous amount of anatomic variability, with cadaveric studies showing that the nerve is located above the lingual aspect of the alveolar crest in approximately $7 \%$ of specimens (4). Roughly $26 \%$ of specimens were found to have a lingual nerve in direct contact with the lingual plate (4). Miloro et al. studied the position of the lingual nerve in 10 healthy volunteers based on MRI examinations (5). He found the mean vertical distance of the nerve from the lingual crest to be $2.75 \pm 0.97 \mathrm{~mm}$, with the nerve located above the lingual crest in $10 \%$ of cases (5). The mean horizontal distance of the nerve to the lingual plate was $2.53 \pm 0.67 \mathrm{~mm}$, and the nerve was in direct contact with the lingual plate in $25 \%$ of cases (5). The lingual nerve can be injured in third molar surgery by placing the surgical incision too close to the lingual soft tissues or by haphazard use of a surgical bur resulting in perforation of the lingual plate. Furthermore, there is a significant increase in the incidence of lingual nerve injuries in cases where lingual retraction or tooth sectioning are performed (6). One study found that vertical tooth sectioning was more likely than horizontal sectioning to result in lingual nerve injury, although both techniques were associated with increased risk (6). In this same study, the use of an Obwegeser periosteal elevator rather than a Freer periosteal elevator to raise a lingual flap resulted in increased rates of lingual nerve injury (6). Primary wound closure was not found to be associated with lingual nerve damage (6). The lingual nerve cannot be visualized on routine pre-operative imaging, making it impossible to know its precise location during surgery (7). As such, the surgeon should exercise extreme caution when working near lingual hard and soft tissues (7).

The inferior alveolar nerve, on the other hand, is easily identified on both panoramic and cone beam computed tomography (CBCT), allowing the surgeon to determine the level of risk for nerve injury in advance of surgery. The depth of impaction is directly related to the incidence of nerve injury, and there are characteristic radiographic findings which suggest close proximity of the tooth roots to the inferior alveolar nerve (8). Such findings include darkening of the tooth roots and loss of continuity or deflection of the mandibular canal (9). Intraoperative findings suggestive of likely postoperative paresthesia include profuse bleeding from the surgical site and visualization of the inferior alveolar nerve (10). In cases where it has been determined pre-operatively that there is a high risk of inferior alveolar nerve injury, many practitioners may consider intentional partial odontectomy over extraction. By its very nature, this procedure results in remnant root tips which may secondarily become infected, but it carries significantly less risk of injury to the inferior alveolar nerve in cases of deeply impacted wisdom teeth (11). Patients must be informed that root tip migration may occur over time, necessitating root removal at a later date.

There are documented reports of inferior alveolar nerve block or local infiltration causing permanent paresthesia in the distribution of the inferior alveolar or lingual nerves. Pogrel found that for the inferior alveolar nerve block, the estimated incidence of injury ranges from 1:26,762 to $1: 160,571$ (12). While most of these cases undergo spontaneous recovery, some may result in permanent sensory deficits. Using a needle that is either blunt or barbed due to multiple injections may also cause injury upon withdrawal from the intraoral soft tissues, resulting in an intraneural hematoma and subsequent paresthesia (13). Chemical injury to the nerve due to local anesthetic injection is another possibility, but this is a less likely scenario as the epineurium does not stretch (13). Further investigation is needed to determine the exact mechanism of nerve injury following local anesthetic injection.

Placement of endosseous dental implants has increased dramatically over the past several decades due to improvements in technology and patient satisfaction with such treatments. However, altered sensation in the distribution of the inferior alveolar or mental nerves is an untoward event which may result if anatomic boundaries are not respected during implant placement. For example, the anterior loop of the mental nerve runs up to $5 \mathrm{~mm}$ anterior to the radiographic limit of the mental foramen (14). Additionally, the posterior edentulous mandible often exhibits some degree of vertical bone atrophy, making this region particularly susceptible to iatrogenic nerve injury. While direct trauma of the nerve by an implant drill may occur, other causes of altered sensation post-operatively 


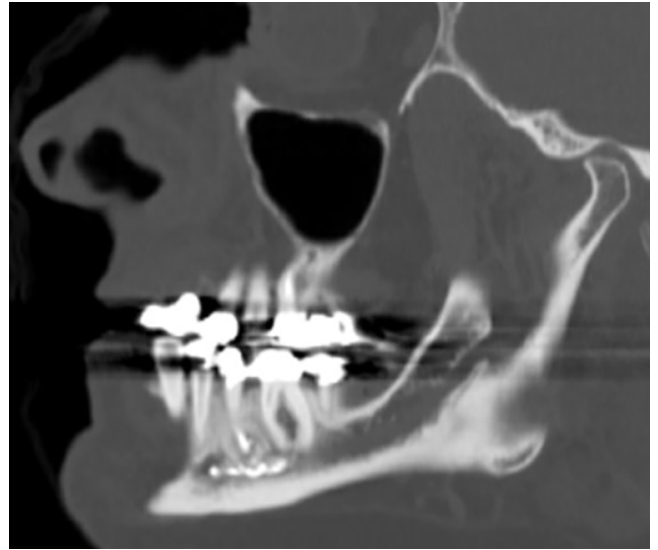

Figure 1 This patient was referred following an endodontic injury where root canal filling cement extruded into the mandibular canal. Findings of violation of the mandibular canal are seen on CT imaging as shown above.

include bleeding within the mandibular canal, thermal injury due to overheating of the bone and surrounding structures, and compression of the nerve due to edema (15). As such, it is recommended that endosseous dental implants be placed a safe distance of $2 \mathrm{~mm}$ away from neurovascular structures in the mandible (16). In cases of a severely atrophic mandible where there is inadequate height for implant placement, the use of short dental implants ( $\leq 6 \mathrm{~mm}$ in height) has been proposed as a useful alternative. Favorable long-term results employing this technique are lacking in the literature (17). One can also plan the implant based on pre-operative CBCT imaging to remain either buccal or lingual to the inferior alveolar nerve. However, this relies upon adequate width of bone for safe placement and is often difficult to execute surgically without the aid of a guide or navigation system (18). Lastly, nerve lateralization techniques may be employed prior to implant placement, although this technique invariably causes some degree of permanent sensory disturbance due to manipulation of the nerve at the time of surgery (19). The majority of patients will have resolution of these symptoms within 6 months, but the invasive nature of this procedure limits its use today (19).

Endodontic treatment can also result in injuries to the inferior alveolar nerve. Such injuries are often the result of overzealous instrumentation of mandibular molars and perforation of the root apices, thereby allowing a direct pathway for chemical or mechanical irritants to enter the mandibular canal (20) (Figure 1). Chemical injury may result in fibrosis of the nerve, or an exophytic neuroma may develop if the epineurium is violated (21).

Iatrogenic trauma to the inferior alveolar nerve may also be observed during orthognathic surgery, but many consider this to be an expected outcome rather than a complication due to the intimate relationship between the affected nerve branches and the planned osteotomies. The bilateral sagittal split osteotomy (BSSO) usually results in temporary postoperative paresthesia of the inferior alveolar nerve, as the nerve is visualized and manipulated during surgery (22). The lingual nerve is less commonly at risk, but excessive lingual retraction or placement of long screws perforating the lingual cortex may result in temporary sensory disturbances (22). Genioplasty employs a mandibular vestibular incision to dissect and expose the mental nerves bilaterally prior to making an osteotomy. These nerves may be inadvertently injured during the dissection or if the osteotomy is placed too close to the anterior loop of the mental nerve (23). Most neurosensory disturbances caused by retraction during genioplasty procedures undergo spontaneous recovery (23). Similarly, the infraorbital nerves are exposed when performing a LeFort I osteotomy, and the pressure placed on this nerve by retraction often results in a temporary hypoesthesia to the upper lip, cheeks, lower eyelids, and lateral aspects of the nose following surgery (24).

Temporomandibular joint (TMJ) arthroscopy is an uncommon cause of trigeminal nerve injury, with the auriculotemporal nerve being most at risk. Up to $23.4 \%$ of patients sustain a temporary paresthesia over the distribution of this nerve which can be attributed to compression from increased hydrostatic pressure or fluid extravasation when irrigating the joint space (25). A more severe complication of TMJ arthroscopy involves perforation of the medial joint capsule with resultant extravasation of fluid into the pterygomandibular space, thereby compressing the inferior alveolar and lingual nerves which is also generally selflimiting (25).

Maxillofacial trauma is responsible for a significant portion of the trigeminal nerve injuries seen by the oral and maxillofacial surgeon. The inferior alveolar nerve is susceptible to injury during mandibular trauma, as parasymphyseal fractures may propagate through the mental foramen causing permanent paresthesia (26). Likewise, a displaced mandibular body or angle fracture may cause shearing or traction injury to the inferior alveolar nerve (26). Gunshot wounds to the mandible are highly destructive and, depending on their location, may transect or irreversibly traumatize the inferior alveolar nerve (27). Zygomaticomaxillary complex (ZMC) fractures consist of 
a fracture of the inferior orbital rim which often extends through the infraorbital foramen and canal, affecting the infraorbital nerve (28). A frontal bone fracture overlying the supraorbital foramen may damage the supraorbital nerve in a similar fashion (29).

Surgical repair of facial fractures is another way in which the various branches of the trigeminal nerve may be injured. For example, nerves may be impaled by drill bits and screws used for rigid fixation of fracture segments (30). Nerve damage may also occur when manipulating fracture segments or during dissection and exposure of fractures (30). As such, a thorough examination of the trauma patient is required prior to surgery, and any sensory disturbances must be documented for medicolegal purposes. In most cases, nerve decompression is facilitated by appropriate reduction and fixation of fractures (2). Potential sensory complications may be limited by employing monocortical plating techniques that avoid penetration of the mandibular canal with screws (2). Additionally, careful placement of any bicortical hardware at the inferior mandibular border can prevent iatrogenic injury to the inferior alveolar nerve.

Finally, patients may present with trigeminal nerve injuries stemming from locally destructive disease processes including malignancy (i.e., sarcomas, jaw tumors, and salivary gland lesions) (31). Paresthesia of multiple divisions of the trigeminal nerve is characteristic of an underlying malignancy or a neurodegenerative process such as multiple sclerosis (31). Central cortical lesions from a prior cerebrovascular accident may contribute to trigeminal nerve sensory deficits as well (31). We present the following article in accordance with the Narrative Review reporting checklist (available at https://fomm.amegroups.com/article/ view/10.21037/fomm-21-26/rc).

\section{Methods}

\section{Classification of trigeminal nerve injuries}

A thorough literature review was conducted to determine the most frequently used classification system for nerve injuries. Additionally, methods for performing clinical neurosensory testing and treating nerve injuries were investigated using online databases such as PubMed and MEDLINE. The Medical Research Council Scale (MRCS) is commonly used to characterize trigeminal nerve injuries and their subsequent recovery. This scale has eight different categories ranging from S0 (no recovery) to S4 (full recovery) and is based on clinical assessments of sensory function (32) (table detailing the MRCS scale can be found in article \#44 from references section by Bagheri et al.). Using the MRCS system, functional sensory recovery (FSR) is defined as a rating of $\mathrm{S} 3$ or greater (32). $\mathrm{S} 3$ corresponds to recovery of pain and touch sensation without over-response and static 2-point discrimination less than $15 \mathrm{~mm}$ (32). Other classification systems used to assess recovery of sensory function include the Seddon and Sunderland systems. Both systems characterize nerve injuries based on the depth of injury (i.e., whether the injury extends to the level of the epineurium, perineurium, or endoneurium) (33). According to Seddon, there are three categories of injury, which are referred to as neuropraxia, axonotmesis, and neurotmesis (listed in order of increasing severity) (33). Sunderland's classification describes two additional categories of injury depending on the extent of damage to the fascicular nerve structure (33).

\section{Clinical neurosensory testing}

A standardized method of neurosensory testing is critical to accurately group patients into one of the aforementioned categories and to monitor for potential clinical improvement over time. A typical examination will begin with a clinical mapping of the face using a soft brush stroke to delineate the areas of sensory disturbance. Next, static light touch and pressure conveyed by the A-beta nerve fibers are evaluated using Von Frey monofilaments $(34,35)$. During testing, the monofilaments are aligned perpendicular to the skin surface and pressed against the area of interest until the filament begins to bend (Figure 2A) (36). Each filament bends at a specific applied force (36). The patient alerts the clinician when they feel light touch on their face, and the smallest detectable filament size is recorded. Normal trigeminal nerve function is characterized by detection of either of the two smallest monofilaments (1.65 or 2.36) (36).

Directional discrimination is performed using either a fine brush or a Von Frey monofilament and is done to assess functioning of the large, myelinated A-alpha and A-beta fibers (36). A two-point discrimination test is completed using a boley gauge or other comparable device to assess the A-delta and unmyelinated C-fibers (Figure 2B) (36). The two points are initially placed far apart and then brought closer to one another in $2 \mathrm{~mm}$ increments until the patient can no longer detect separate points (36). Normal two-point discrimination is 4 to $5 \mathrm{~mm}$ for the inferior alveolar nerve and 3 to $4 \mathrm{~mm}$ for the lingual nerve (36).

Pin pressure nociception evaluates the free nerve 

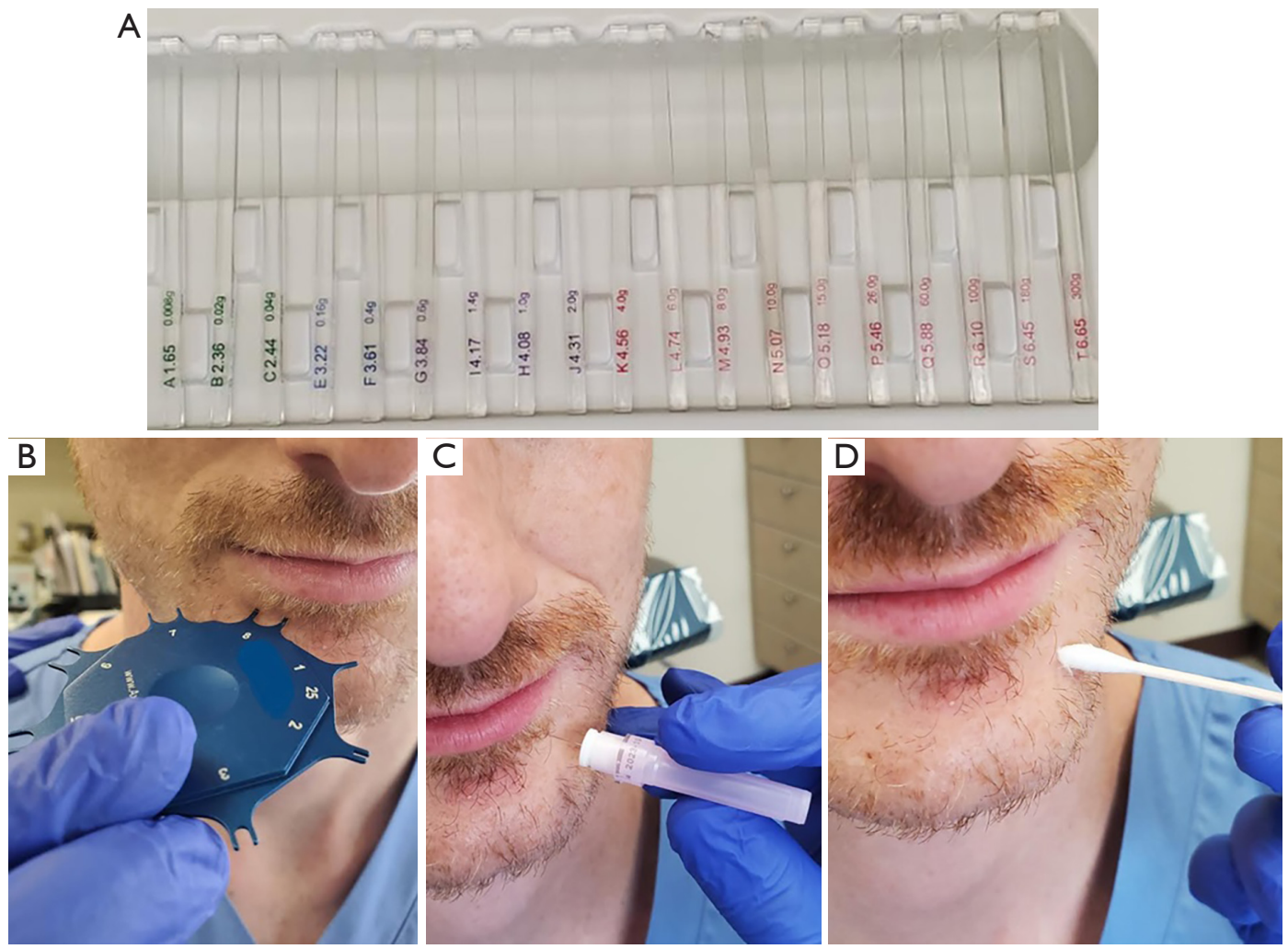

Figure 2 Clinical neurosensory testing is undertaken to correctly diagnose a trigeminal nerve injury. (A) As part of neurosensory testing, Von Frey monofilaments are placed against the affected skin or mucosal surface. Each monofilament bends at a specific applied force. (B) Twopoint discrimination is assessed using a device such as the one depicted in the image. (C) Pin pressure nociception is evaluated by applying pressure with a dental needle to the affected area of the face. (D) Response to thermal stimuli is assessed by applying Coltene Endo-ice to a cotton tip applicator which is then placed against the affected area.

endings supplied by poorly myelinated A-delta and unmyelinated C-fibers (34). A sterile dental needle is applied to the affected area of the face in a pinprick fashion, and the patient comments on whether the intensity of the applied stimulus is increased, decreased or the same as the normal side (Figure 2C) (36). Cold stimuli perceived by unmyelinated C-fibers can be tested using Endo-Ice (Coltene) sprayed on a cotton tip applicator (Figure 2D) (36). Heat sensation, carried by the A-delta fibers, is assessed using a cotton tip applicator placed in hot water (36).

\section{Discussion}

\section{Trigeminal nerve microsurgery: indications}

There are specific indications for trigeminal nerve microsurgery, and only a small percentage of patients who are evaluated for a trigeminal nerve injury will eventually undergo surgery. In order to be considered a surgical candidate, the neurosensory disturbance must persist for more than three months, thus ensuring that spontaneous recovery is unlikely (37). Other indications for surgery include lack of sensory improvement at three months as well as the development of dysesthesia (37). Worsening hypoesthesia and hypoesthesia that is poorly tolerated by the patient are also indications for surgery (37). Patients with observed nerve transections are immediate surgical candidates, as the degree of injury is visualized and there is no chance for achieving FSR in such cases without reanastomosis of the severed nerve stumps (37). Use of magnetic resonance neurography may demonstrate a complete nerve injury (38). Contraindications to trigeminal nerve microsurgery include central neuropathic pain, improving sensory function, hypoesthesia which is tolerated well by the patient, and excessive time since injury (39). Furthermore, surgery is contraindicated in patients with 

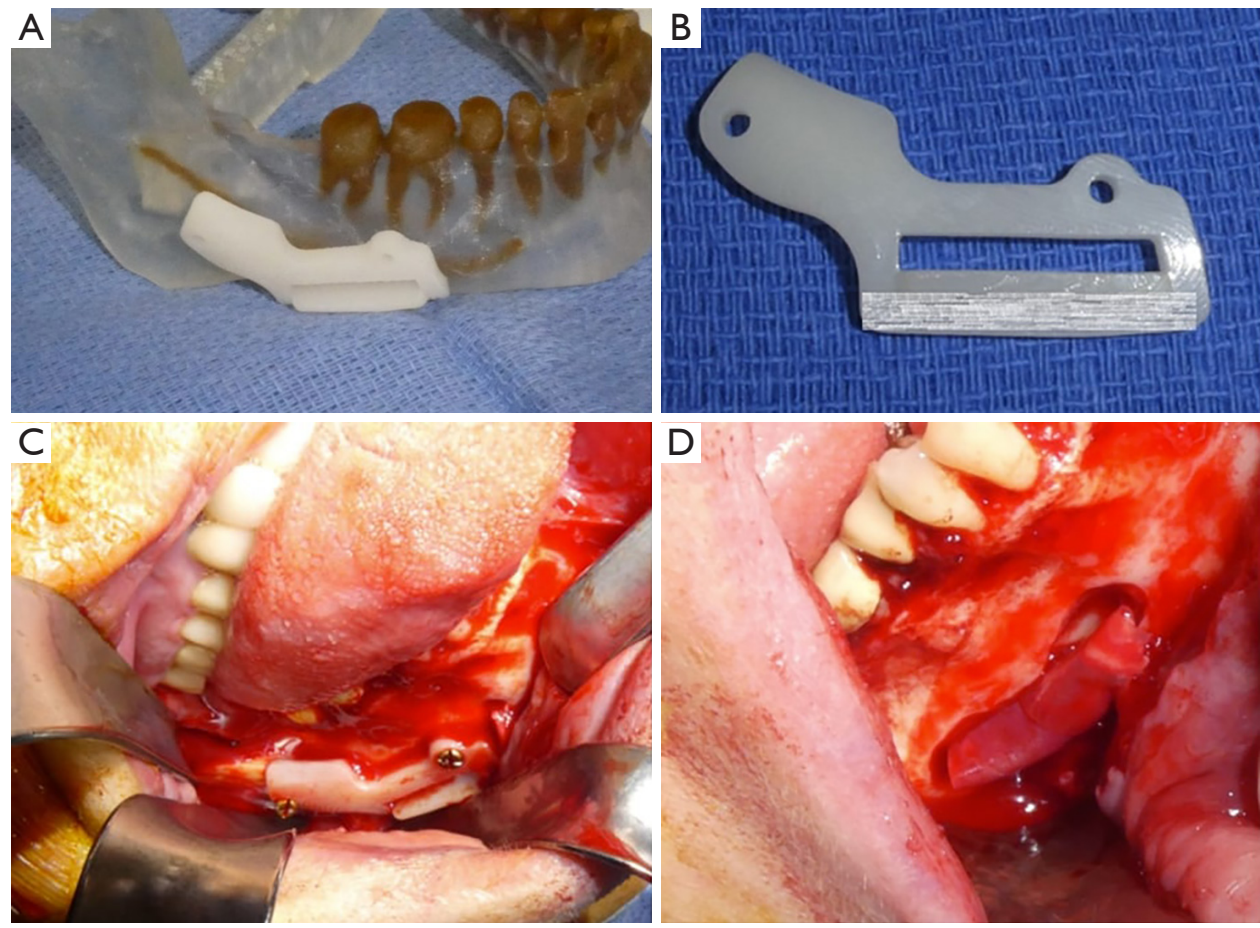

Figure 3 This case demonstrates inferior alveolar nerve repair following iatrogenic injury due to extruded endodontic material. (A) Surgical model showing area of planned cortical bone removal to access the injured nerve. (B) Virtually planned guide which is sterilized prior to surgery. (C) Following exposure of the lateral mandible, the surgical guide is adapted to the lateral border of the mandible using fixation screws. (D) Following decortication of the lateral mandible, all foreign material and non-viable nerve tissue were removed. Due to the size

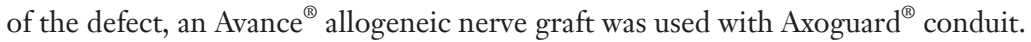

significant medical comorbidities, as they may not be able to safely undergo general anesthesia (39).

\section{Trigeminal nerve microsurgery}

Repair of trigeminal nerve injuries is performed in the operating room setting with the patient under general anesthesia and nasally intubated. A mouth prop and tongue retractor are utilized to allow clear visualization of the retromolar region on the side of injury. In the case of a lingual nerve injury, a sulcular incision is made to approximately the first premolar on the affected side along with a standard third molar incision made posteriorly across the external oblique ridge. A full thickness mucoperiosteal flap is elevated, including on the lingual aspect of the mandible. At this point, 2-0 silk sutures can be placed through the lingual mucosa and sutured to the contralateral side to aid in retraction. A Dierks dissector can then be used to dissect through periosteum on the underside of the lingual flap and to ultimately identify the proximal and distal ends of the lingual nerve.

A similar approach is undertaken in the case of an inferior alveolar nerve repair, but lingual soft tissue elevation and retraction are not necessary. Virtual surgical planning can be used to design a surgical guide for bone removal intraoperatively (Figure 3A,B). Use of a computer generated surgical guide limits the amount of bone removal required and thus minimizes potential complications. Once the lateral posterior mandible is exposed, a round carbide bur is used under saline irrigation to decorticate the cortex overlying the mandibular canal (Figure 3C,D). When in close proximity to the canal, a less aggressive round diamond bur is used to avoid inadvertent trauma to the nerve. Following complete exposure of the damaged nerve, microsurgical repair may ensue. Trigeminal nerve repair is most commonly completed at the level of the epineurium, as there are no observed differences in clinical outcomes between epineural and perineural repairs (39). A 7-0 or smaller diameter suture is selected in a non-inflammatory material such as nylon to limit post-operative scar formation (39). 


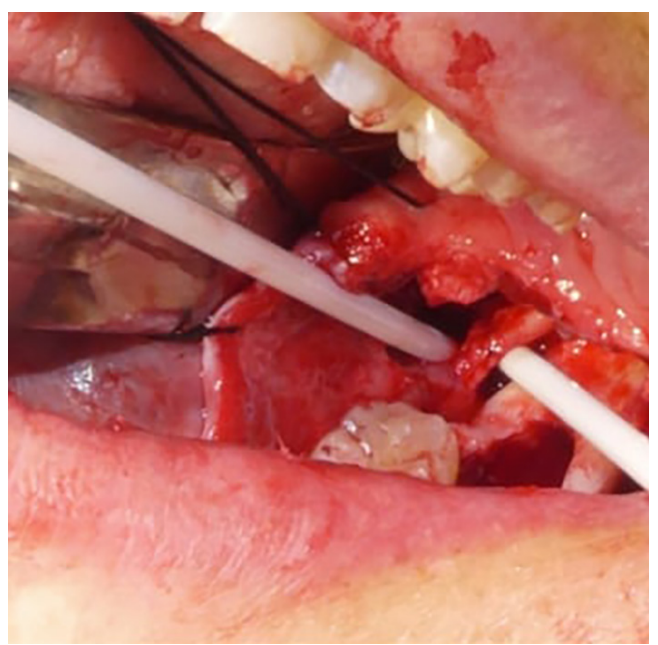

Figure 4 This patient sustained a complete lingual nerve injury following third molar removal. Excision of neuroma and primary neurorrhaphy were performed in this case as depicted in the image.

Sutures placed across the epineurium must be secured in a tension-free manner. If primary, tension-free repair is not possible, use of a nerve graft is indicated. Alternatively, conduit guided repairs can be performed to minimize tension at the repair site.

External neurolysis is the process of freeing a nerve from its surrounding tissue bed and removing any adherent scar tissue which may interfere with examination and eventual nerve recovery (39). In the case of a patient with a moderate sensory disturbance, removing scar tissue from the intact nerve and decompressing the nerve may be the only procedures necessary to promote sensory recovery (39). Any foreign bodies encroaching upon the nerve, including endodontic filling material, tooth remnants, or implant materials must also be removed at this time (Figure 3D) (39).

Internal neurolysis is rarely required in cases where there is evidence of nerve fibrosis. This technique uses an incision along the epineurium to expose the internal fascicles (epifascicular epineurotomy). Releasing the epineural fibrosis in this manner ideally results in expansion and decompression of the nerve. In cases that are refractory to internal neurolysis techniques, the affected segment should be excised in its entirety and the resultant defect repaired via primary neurorrhaphy or nerve graft (39).

Neuromas are excised when present, leaving behind proximal and distal nerve segments which are then examined for the presence of herniated interfascicular tissues which are suggestive of normal tissue. Once healthy nerve tissue is identified on each end, the two nerve stumps are coapted using approximately 3-4 nylon sutures placed along the epineurium (Figure 4) (39). If the nerve cannot be re-approximated in a tension-free manner, mobilization of the nerve is attempted by bluntly dissecting around the proximal and distal stumps. A continuity defect may still exist despite nerve mobilization techniques, necessitating a nerve grafting procedure. Historically, grafting procedures for trigeminal nerve injuries involved the use of autogenous donor nerves, most commonly the sural and great auricular nerves due to their ease of harvest and low overall morbidity (40). However, there are often discrepancies in nerve diameter and number of nerve fascicles between the aforementioned donor nerves and the trigeminal nerve (41). Such differences can contribute to a decreased likelihood of successful nerve regeneration and recovery of sensory function (41). An alternative approach to treating continuity defects of the trigeminal nerve is by using allogeneic nerve grafts. Currently, Avance ${ }^{\circledR}$ (Axogen, Alachua, Florida) is the only FDA approved human nerve allograft (42). The potential benefits of an allogeneic graft over autograft include no need for a donor site harvest, decreased operating room time, and larger possible lengths of nerve graft. The graft is secured to the proximal and distal nerve stumps in a similar fashion to the epineural repair described previously (Figures 3D, 5A). A nerve conduit can then be laid over the coapted nerve ends to isolate and protect the repair throughout the healing process. A fibrin-based glue is used to hold the nerve conduit in place (Figure 5B). Commonly used nerve conduits include collagen tubules such as NeuraGen ${ }^{\circledR}$ (Integra LifeSciences Corporation, Plainsboro, NJ) and Axoguard ${ }^{\circledR}$ (Axogen, Alachua, Florida).

\section{Trigeminal nerve microsurgery: outcomes}

There are limited studies documenting the outcomes of trigeminal nerve microsurgeries, with most available data from case reports and case series. In a study examining patients referred for lingual and inferior alveolar nerve injuries over a 5 -year period, Pogrel found that surgery produced slightly better results in the inferior alveolar nerve group compared to the lingual nerve group (43). Early repairs (performed before 10 weeks post-injury) were more successful than those performed later (43). No patients in the study developed a post-operative dysesthesia if they did not have this condition pre-operatively (43). Pogrel went on to conclude that trigeminal nerve microsurgery, if performed when indicated, can provide improved sensation 

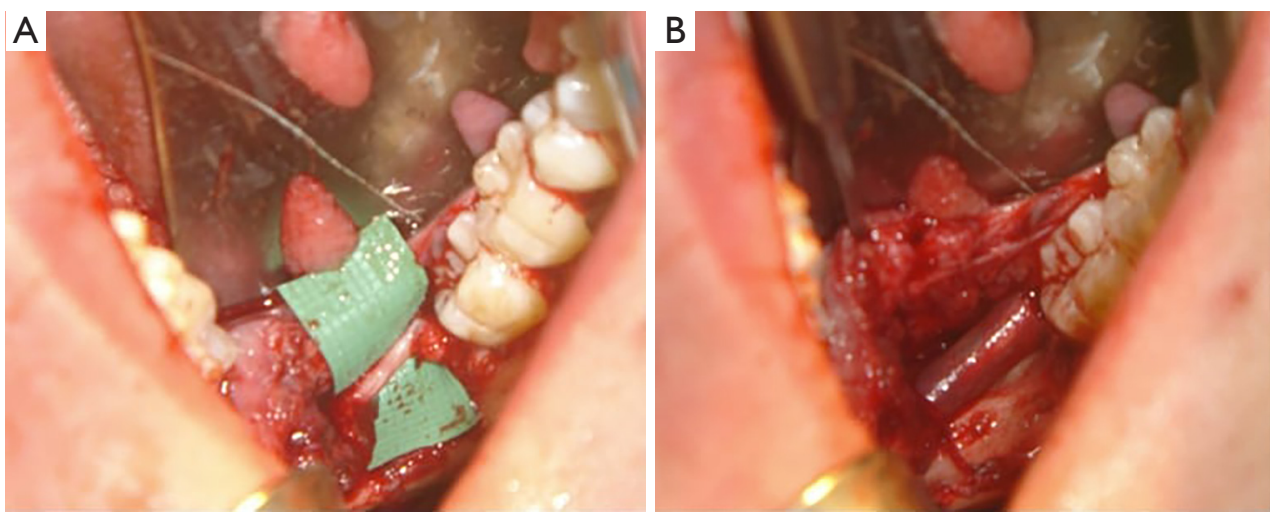

Figure 5 This patient sustained a complete lingual nerve injury following third molar removal and thus required surgical intervention. (A) The resultant defect was too large for primary neurorrhaphy, so an Avance ${ }^{\circledR}$ allogeneic interpositional graft was placed. (B) Axoguard ${ }^{\circledR}$ conduit used for entubulization of the nerve

in greater than $50 \%$ of patients (43). A similar retrospective cohort study performed by Bagheri et al. examined FSR in 167 individuals following microsurgical repair of the inferior alveolar nerve (44). In this cohort, FSR was achieved in $88.9 \%$ of patients who underwent primary neurorrhaphy, $85 \%$ of patients who underwent external decompression, $75 \%$ of patients who had internal neurolysis, and $70.6 \%$ of patients who had excision of an exophytic neuroma without additional treatments (44). Bagheri also performed a retrospective chart review of 222 patients with lingual nerve injuries who underwent subsequent surgical repair via various techniques such as internal neurolysis, neurorrhaphy, or autogenous nerve graft placement (32). He found that $90.5 \%$ of patients achieved FSR of S3 or greater following microsurgery, with only $9.5 \%$ of patients failing to improve (32). Studies examining indirect repairs using nerve allografts have shown promising results as well. A recent study evaluating eight patients with lingual nerve injuries and eight patients with inferior alveolar nerve injuries treated with allogeneic nerve grafts found that all but one patient achieved functional sensory recovery during the follow up period (45).

Recently, the use of platelet-rich plasma (PRP) during trigeminal nerve microsurgery has been proposed as a means of improving nerve recovery. PRP has been used for many years in other oral surgery procedures such as jaw reconstruction and dental implant surgery with successful outcomes. It is obtained by centrifuging the patient's own blood in such a way that the red blood cells are removed, leaving behind platelets rich in growth factors and proteins (46). Upon degranulation of platelet alpha- granules, many of these growth-promoting molecules are released into the surrounding environment where they act to promote tissue repair (46). There are numerous studies supporting the use of PRP for enhanced soft tissue healing, but limited data exists regarding its effect on peripheral nerve regeneration. In a study on this topic using an animal model, Cho et al. transected the facial nerve of 20 albino guinea pigs and then performed subsequent nerve repair, either with sutures only or PRP in addition to sutures (47). Guinea pigs treated with perineural sutures and PRP had statistically significant improvement in functional and electrophysiological outcomes compared to the suture only control group at 4 and 6 weeks post-repair (47). Upon histological analysis, nerves repaired with perineural sutures and PRP showed a significant increase in the number of myelinated axons relative to the control group (47). Based on these initial results, the use of PRP and other similar biologic therapies in peripheral nerve repair warrants further investigation in human subjects. Should additional published data on surgical outcomes become available, treatment recommendations will likely be updated, as the field of trigeminal nerve microsurgery is still in its infancy.

\section{Acknowledgments}

Funding: None.

\section{Footnote}

Reporting Checklist: The authors have completed the Narrative Review reporting checklist. Available at https://fomm. 
amegroups.com/article/view/10.21037/fomm-21-26/rc

Peer Review File: Available at https://fomm.amegroups.com/ article/view/10.21037/fomm-21-26/prf

Conflicts of Interest: Both authors have completed the ICMJE uniform disclosure form (available at https://fomm. amegroups.com/article/view/10.21037/fomm-21-26/ coif). VBZ serves as an unpaid editorial board member of Frontiers of Oral and Maxillofacial Medicine from Nov 2019 to Oct 2021. VBZ serves as consultant for Axogen, Alachua, FL. The other author has no conflict of interest to declare.

Ethical Statement: The authors are accountable for all aspects of the work in ensuring that questions related to the accuracy or integrity of any part of the work are appropriately investigated and resolved.

Open Access Statement: This is an Open Access article distributed in accordance with the Creative Commons Attribution-NonCommercial-NoDerivs 4.0 International License (CC BY-NC-ND 4.0), which permits the noncommercial replication and distribution of the article with the strict proviso that no changes or edits are made and the original work is properly cited (including links to both the formal publication through the relevant DOI and the license). See: https://creativecommons.org/licenses/by-nc-nd/4.0/.

\section{References}

1. Frequency of trigeminal nerve injuries following third molar removal. Br Dent J 2005;199:579.

2. Ziccardi VB, Assael LA. Mechanisms of trigeminal nerve injuries. Atlas Oral Maxillofac Surg Clin North Am 2001;9:1-11.

3. Meshram VS, Meshram PV, Lambade P. Assessment of nerve injuries after surgical removal of mandibular third molar: a prospective study. Asian J Neurosci 2013;2013. doi: 10.1155/2013/291926

4. Dias GJ, de Silva RK, Shah T, et al. Multivariate assessment of site of lingual nerve. Br J Oral Maxillofac Surg 2015;53:347-51.

5. Miloro M, Halkias LE, Slone HW, et al. Assessment of the lingual nerve in the third molar region using magnetic resonance imaging. J Oral Maxillofac Surg 1997;55:134-7. Burian E, Probst FA, Weidlich D, et al. MRI of the inferior alveolar nerve and lingual nerve-anatomical variation and morphometric benchmark values of nerve diameters in healthy subjects. Clin Oral Investig 2020;24:2625-34.

6. Valmaseda-Castellón E, Berini-Aytés L, Gay-Escoda C. Lingual nerve damage after third lower molar surgical extraction. Oral Surg Oral Med Oral Pathol Oral Radiol Endod 2000;90:567-73.

7. Burian E, Probst FA, Weidlich D, et al. MRI of the inferior alveolar nerve and lingual nerve-anatomical variation and morphometric benchmark values of nerve diameters in healthy subjects. Clin Oral Investig 2020;24:2625-34.

8. Lata J, Tiwari AK. Incidence of lingual nerve paraesthesia following mandibular third molar surgery. Natl J Maxillofac Surg 2011;2:137-40.

9. Szalma J, Lempel E, Jeges S, et al. Darkening of third molar roots: panoramic radiographic associations with inferior alveolar nerve exposure. J Oral Maxillofac Surg 2011;69:1544-9.

10. Sarikov R, Juodzbalys G. Inferior alveolar nerve injury after mandibular third molar extraction: a literature review. J Oral Maxillofac Res 2014;5:e1.

11. Martin A, Perinetti G, Costantinides F, et al. Coronectomy as a surgical approach to impacted mandibular third molars: a systematic review. Head Face Med 2015;11:9.

12. Pogrel MA, Thamby S. Permanent nerve involvement resulting from inferior alveolar nerve blocks. J Am Dent Assoc 2000;131:901-7.

13. Ozen T, Orhan K, Gorur I, et al. Efficacy of low level laser therapy on neurosensory recovery after injury to the inferior alveolar nerve. Head Face Med 2006;2:3.

14. Iyengar AR, Patil S, Nagesh KS, et al. Detection of anterior loop and other patterns of entry of mental nerve into the mental foramen: A radiographic study in panoramic images. J Dent Implant 2013;3:21-5.

15. Juodzbalys G, Wang HL, Sabalys G. Injury of the Inferior Alveolar Nerve during Implant Placement: a Literature Review. J Oral Maxillofac Res 2011;2:e1.

16. Renton T. Prevention of iatrogenic inferior alveolar nerve injuries in relation to dental procedures. Dent Update 2010;37:350-2, 354-6, 358-60 passim.

17. Papaspyridakos P, De Souza A, Vazouras K, et al. Survival rates of short dental implants $(\leq 6 \mathrm{~mm})$ compared with implants longer than $6 \mathrm{~mm}$ in posterior jaw areas: A metaanalysis. Clin Oral Implants Res 2018;29 Suppl 16:8-20.

18. Chen YT, Chiu YW, Peng CY. Preservation of Inferior Alveolar Nerve Using the Dynamic Dental Implant Navigation System. J Oral Maxillofac Surg 2020;78:678-9.

19. Abayev B, Juodzbalys G. Inferior alveolar nerve lateralization and transposition for dental implant placement. Part I: a systematic review of surgical 
techniques. J Oral Maxillofac Res 2015;6:e2.

20. Dempf R, Hausamen JE. Lesions of the inferior alveolar nerve arising from endodontic treatment. Aust Endod J 2000;26:67-71.

21. Marques TM, Gomes JM. Decompression of inferior alveolar nerve: case report. J Can Dent Assoc 2011;77:b34.

22. Hanzelka T, Foltán R, Pavlíková G, et al. The role of intraoperative positioning of the inferior alveolar nerve on postoperative paresthesia after bilateral sagittal split osteotomy of the mandible: prospective clinical study. Int J Oral Maxillofac Surg 2011;40:901-6.

23. Ousterhout DK. Sliding genioplasty, avoiding mental nerve injuries. J Craniofac Surg 1996;7:297-8.

24. Garg S, Kaur S. Evaluation of Post-operative Complication Rate of Le Fort I Osteotomy: A Retrospective and Prospective Study. J Maxillofac Oral Surg 2014;13:120-7.

25. Weinberg S, Kryshtalskyj B. Analysis of facial and trigeminal nerve function after arthroscopic surgery of the temporomandibular joint. J Oral Maxillofac Surg 1996;54:40-3; discussion 43-4.

26. Schenkel JS, Jacobsen C, Rostetter C, et al. Inferior alveolar nerve function after open reduction and internal fixation of mandibular fractures. J Craniomaxillofac Surg 2016;44:743-8.

27. Amole O, Osunde O, Akhiwu B, et al. A 14-Year Review of Craniomaxillofacial Gunshot Wounds in a ResourceLimited Setting. Craniomaxillofac Trauma Reconstr 2017;10:130-7.

28. Westermark A, Jensen J, Sindet-Pedersen S. Zygomatic fractures and infraorbital nerve disturbances. Miniplate osteosynthesis vs. other treatment modalities. Oral Surg Oral Diagn 1992;3:27-30.

29. Strong EB. Frontal sinus fractures: current concepts. Craniomaxillofac Trauma Reconstr 2009;2:161-75.

30. Song Q, Li S, Patil PM. Inferior alveolar and mental nerve injuries associated with open reduction and internal fixation of mandibular fractures: a Seven Year retrospective study. J Craniomaxillofac Surg 2014;42:1378-81.

31. Majoie CB, Verbeeten B Jr, Dol JA, et al. Trigeminal neuropathy: evaluation with MR imaging. Radiographics 1995;15:795-811.

32. Bagheri SC, Meyer RA, Khan HA, et al. Retrospective review of microsurgical repair of 222 lingual nerve injuries. J Oral Maxillofac Surg 2010;68:715-23.

33. Chhabra A, Ahlawat S, Belzberg A, et al. Peripheral nerve injury grading simplified on MR neurography: As referenced to Seddon and Sunderland classifications. Indian J Radiol Imaging 2014;24:217-24.

34. Uddin Z, MacDermid JC. Quantitative Sensory
Testing in Chronic Musculoskeletal Pain. Pain Med 2016;17:1694-703.

35. Yekta SS, Koch F, Grosjean MB, et al. Analysis of trigeminal nerve disorders after oral and maxillofacial intervention. Head Face Med 2010;6:24.

36. Ziccardi VB, Zuniga JR. Nerve injuries after third molar removal. Oral Maxillofac Surg Clin North Am 2007;19:105-15, vii.

37. Ziccardi VB, Steinberg MJ. Timing of trigeminal nerve microsurgery: a review of the literature. J Oral Maxillofac Surg 2007;65:1341-5.

38. Zuniga JR, Mistry C, Tikhonov I, et al. Magnetic Resonance Neurography of Traumatic and Nontraumatic Peripheral Trigeminal Neuropathies. J Oral Maxillofac Surg 2018;76:725-36.

39. Ziccardi VB. Microsurgical techniques for repair of the inferior alveolar and lingual nerves. Atlas Oral Maxillofac Surg Clin North Am 2011;19:79-90.

40. Wolford LM, Stevao EL. Considerations in nerve repair. Proc (Bayl Univ Med Cent) 2003;16:152-6.

41. Jones RH. Repair of the trigeminal nerve: a review. Aust Dent J 2010;5 5:112-9.

42. Kornfeld T, Vogt PM, Radtke C. Nerve grafting for peripheral nerve injuries with extended defect sizes. Wien Med Wochenschr 2019;169:240-51.

43. Pogrel MA. The results of microneurosurgery of the inferior alveolar and lingual nerve. J Oral Maxillofac Surg 2002;60:485-9.

44. Bagheri SC, Meyer RA, Cho SH, et al. Microsurgical repair of the inferior alveolar nerve: success rate and factors that adversely affect outcome. J Oral Maxillofac Surg 2012;70:1978-90.

45. Yampolsky A, Ziccardi V, Chuang SK. Efficacy of Acellular Nerve Allografts in Trigeminal Nerve Reconstruction. J Oral Maxillofac Surg 2017;75:2230-4.

46. Yu W, Wang J, Yin J. Platelet-rich plasma: a promising product for treatment of peripheral nerve regeneration after nerve injury. Int J Neurosci 2011;121:176-80.

47. Cho HH, Jang S, Lee SC, et al. Effect of neural-induced mesenchymal stem cells and platelet-rich plasma on facial nerve regeneration in an acute nerve injury model. Laryngoscope 2010;120:907-13.

doi: $10.21037 /$ fomm-21-26

Cite this article as: Schiavone M, Ziccardi VB. Trigeminal nerve injuries in oral and maxillofacial surgery: a literature review. Front Oral Maxillofac Med 2021;3:28. 